\title{
Myocyte apoptosis occurs early during the development of pressure-overload hypertrophy in infant myocardium
}

\author{
Yeong-Hoon Choi, MD, ${ }^{\text {a,b,d }}$ Douglas B. Cowan, PhD, ${ }^{\mathrm{a}, \mathrm{d}}$ Adrian M. Moran, MBBS, ${ }^{\mathrm{c}}$ Steven D. Colan, MD, ${ }^{\mathrm{c}}$ Christof Stamm, MD, \\ Koh Takeuchi, MD, ${ }^{\mathrm{b}}$ Ingeborg Friehs, MD, ${ }^{\mathrm{b}}$ Pedro J. del Nido, MD, ${ }^{\mathrm{b}}$ and Francis X. McGowan, Jr, MD ${ }^{\mathrm{a}, \mathrm{d}}$
}

\begin{abstract}
Objective: Abnormal hemodynamic loading often accompanies congenital heart disease both before and after surgical repair. Adaptive and maladaptive myocardial responses to increased load are numerous. This study examined the hypothesis that myocyte loss occurs during compensatory hypertrophic growth in the developing infant myocardium subjected to progressive pressure overload.
\end{abstract}

\begin{abstract}
Methods: Pressure-overload left ventricular hypertrophy was induced in 7- to 10-day-old rabbits by banding the thoracic aorta. Left ventricular function and mechanics were quantified by serial echocardiography and noninvasive left ventricular wall stress analysis. Left ventricular tissue sections were examined for fibrosis by using Masson's trichrome stain and for myocyte apoptosis by using a myocyte-specific DNA fragmentation assay and caspase-3 activation (specific fluorescent substrate).
\end{abstract}

Results: Significant myocyte apoptosis $\left(198 \pm 37 / 10^{6}\right.$ myocytes, $P<.01$ vs control) and caspase- 3 activation were present in early hypertrophy when left ventricular contractility was preserved and compensatory hypertrophy had normalized wall stress. By 6 weeks, multiple indices of left ventricular contractility were reduced, and left ventricular wall stress was increased. Myocyte apoptosis was accelerated (361 $\pm 56 / 10^{6}$ myocytes), caspase- 3 activity further increased, and the estimated total number of left ventricular myocytes was significantly reduced by $18 \% \pm 4 \%$.

Conclusion: In experimental infant left ventricular hypertrophy, myocyte apoptosis is initiated in the face of normalized wall stress and preserved contractility. The ongoing rate of apoptosis causes a measurable decrease in myocyte number that is coincident with the onset of ventricular dysfunction. It thus appears that pressure overload, even at its earliest stages, is not well tolerated by the developing ventricle.

Supplemental material is available online.

Congenital heart disease is commonly associated with abnormal hemodynamic loading (pressure or volume). Because complete structural and hemodynamic correction is frequently impossible, abnormal loading is a persistent problem in many patients. In the setting of sustained pressure overload, ventricular adaptation typically progresses through stages that include compensatory hypertrophy caused by the parallel addition of sarcomeres, which results

From the Departments of Anesthesiology and Perioperative and Pain Medicine, ${ }^{\mathrm{a}}$ Cardiac Surgery, ${ }^{\mathrm{b}}$ Cardiology, ${ }^{\mathrm{c}}$ and the Anesthesia/Critical Care Medicine Research Laboratory, ${ }^{\mathrm{d}}$ Children's Hospital Boston, and Harvard Medical School, Boston, Mass.

Supported in part by National Institutes of Health grants HL-74734 and HL-66186 (to F.X.M.) and HL-46207 (to P.J.dN.).

Received for publication July 1, 2008; revisions received Nov 20, 2008; accepted for publication Dec 22, 2008.

Address for reprints: Yeong-Hoon Choi, MD, Children's Hospital Boston and Harvard Medical School, Department of Cardiac Surgery, 300 Longwood Avenue, Boston, MA 02115. Current address: Heartcenter of the University of Cologne, Department of Cardiothoracic Surgery and Center of Molecular Medicine Cologne, Kerpener Str 62, 50924 Cologne, Germany. (E-mail: yh.choi@uk-koeln.de).

J Thorac Cardiovasc Surg 2009; 137:1356-62

$0022-5223 / \$ 36.00$

Copyright (c) 2009 by The American Association for Thoracic Surgery doi:10.1016/j.jtcvs.2008.12.020 in increased myocyte width and ventricular wall thickness. According to LaPlace's Law, myocardial wall stress can be calculated from the formula (pressure $\times$ radius $) /(2 \times$ wall thickness), and thus an increase in wall thickness can ameliorate increased wall stress caused by an increase in pressure. Because ventricular ejection is directly affected by afterload, it is believed that normalization of systolic stress (afterload) via increasing wall thickness is an important mechanism to maintain systolic performance in the face of increased systolic pressure. Failure to do so results in afterload mismatch where the degree of hypertrophy can no longer compensate for increased afterload. ${ }^{1,2}$

Numerous changes in gene expression and protein synthesis accompany this response. They include alterations in sarcomeric protein isoforms and activity; calcium handling and regulatory proteins (eg, the sarcoplasmic reticular Ca-ATPase, SERCA-2); substrate and energy metabolism; adrenergic, muscarinic, and angiotensin receptor species and activity; growth factor and cytokine production; and stimulation of other pleiotropic signaling pathways, such as calcineurin. ${ }^{2-8}$ Abnormalities in parameters such as extracellular matrix signaling, matrix turnover, coronary blood supply, and angiogenesis have also been described and implicated in both the compensatory and pathologic hypertrophic responses.

The specific molecular mechanisms underlying the failure of continued hypertrophic compensation and the progression of contractile dysfunction are not well understood, 


\section{Abbreviations and Acronyms \\ ESS = end-systolic stress \\ FS = fiberstress \\ $\mathrm{LV}=$ left ventricular \\ $\mathrm{mw}=$ midwall \\ SF = shortening fraction \\ SSI = stress shortening index \\ SVI = stress velocity index \\ TUNEL $=$ terminal deoxynucleotide transferase-mediated dUTP nick-end labeling \\ $\mathrm{VCFc}=$ rate of circumferential fiber shortening}

especially in the immature, developing ventricle. Myocyte apoptosis has been implicated in numerous states associated with reduced myocardial performance, including acute and chronic ischemia, diabetes, myocarditis, Adriamycin toxicity, hypertrophy, and heart failure. ${ }^{8-19}$ Although the data showing that apoptosis occurs in these settings are significant, their functional importance remains unclear, particularly with regard to the adaptation to chronic pressure load. Several studies have quantified cardiomyocyte apoptosis in late-stage chronic hypertrophy of adult myocardium, ${ }^{20,21}$ but there is no evidence of this process in the developing heart. Cell growth, survival, and death signaling pathways are different than in the adult in both the normal developing myocardium and that exposed to pressure overload. We therefore examined the hypothesis that hypertrophic growth would stimulate myocyte apoptosis in the developing ventricle independently from the onset of heart failure.

\section{MATERIALS AND METHODS \\ Animal Model}

The institutional animal care and use committee approved these experiments. New Zealand white rabbits (aged 7-10 days) were used to create a model of progressive left ventricular (LV) hypertrophy. After anesthesia was induced with intramuscular ketamine $(20 \mathrm{mg} / \mathrm{kg})$ and xylazine $(0.5$ $\mathrm{mg} / \mathrm{kg}$ ), a left thoracotomy was performed under sterile conditions. A 2-0 silk suture was placed around the descending aorta just distal to the ligamentum arteriosum, with care taken to make the suture as snug as possible without acutely causing stenosis of the descending aorta. After chest closure, air was evacuated from the left thoracic cavity by catheter aspiration. After recovery from anesthesia, animals were returned to their mothers and allowed to feed in the normal manner. With growth, an aortic coarctation gradually develops. In preparation for later evaluations, weekly shaving of the right upper limb and thorax, as well as sham echocardiograms, were performed to accustom the animals to the procedure and reduce the stress response to actual echocardiography, thus avoiding the need for anesthesia or sedation during serial echocardiographic examinations.

For controls, age- and litter-matched New Zealand white rabbits were sham-operated and otherwise handled identically. Echocardiography and noninvasive blood pressure determination were performed at weekly intervals in the banded and age-matched control animals (see Appendix). A separate group of normal animals was used to determine the normal relationship in the rabbit of shortening fraction (SF) to afterload (end-systolic stress [ESS]) (stress shortening index [SSI]), rate of circumferential fiber shortening to ESS (afterload) (VCFc to ESS; stress velocity index [SVI]), and their midwall equivalents $\left(\mathrm{SF}_{\mathrm{mw}}\right.$ and $\mathrm{VCF}_{\mathrm{mw}}$ ) compared with fiberstress (FS) to determine $\mathrm{SSI}_{\mathrm{mw}}$ and $\mathrm{SVI}_{\mathrm{mw}}$. Blood pressure was determined serially and noninvasively using optical plethysmography (Finapress, Ohmeda, Englewood, Colo). The Finapress was applied to the shaved right forearm after application of topical alcohol over the region of the palpable brachial artery. The accuracy of the Finapress versus direct central arterial cannulation (carotid artery) to measure arterial blood pressure and calculate load-adjusted echocardiographic indices was validated in a separate set of experiments (see Appendix for details).

\section{Histochemical Methods}

Animals were sacrificed at weekly intervals, and hearts were immediately perfusion fixed in $4 \%$ paraformaldehyde in phosphate-buffered saline, $\mathrm{pH} 7.4$, and paraffin embedded and sectioned. Mid-LV myocardial sections were deparaffinized, hydrated in a descending alcohol series, and boiled 3 times for 5 minutes in $1 \mathrm{mmol} / \mathrm{L}$ EDTA pH 8.0 (antigen retrieval) before staining. Slides were imaged on a Zeiss Axiovert microscope (Carl Zeiss MicroImaging, Inc., Thornwood, NY) equipped with visible/ultraviolet/ fluorescent objectives $(4-100 \times)$, xenon light source/Sutter filter wheel (Sutter Instrument Company, Novato, Calif), and appropriate excitation/ emission filter sets. Images were recorded using a Princeton Instruments cooled CCD camera (Princeton Instruments, Trenton, NJ) or Leica digital color camera (Leica Microsystems Inc., Bannockburn, Ill), depending on the probe type; images were stored and analyzed using MetaMorph and MetaFluor software (Molecular Devices, Sunnyvale, Calif).

\section{Quantification of Apoptosis}

Terminal deoxynucleotide transferase-mediated dUTP nick-end labeling (TUNEL) staining of DNA stand breaks was done with enhanced fluorescent detection by labeling cleaved double-stranded DNA in tissue sections following the Fluorescein FragEL detection kit (Oncogene, Boston, Mass). Positive and negative controls of this assay were previously performed in in vitro cell culture models using rabbit thoracic lymph nodes (data not shown). For DNA staining of nuclei, $10 \mathrm{mg} / \mathrm{mL}$ Hoechst 33258 in phosphate-buffered saline $/ 50 \%$ glycerol was used. Myocytes were identified by co-staining with mouse anti-desmin monoclonal antibody (Sigma, St Louis, Mo) at a dilution of 1:100 in phosphate-buffered saline containing 5\% horse serum and detected with Alexa 568 goat anti-mouse secondary antibody (Molecular Probes, Eugene, Ore). By using MetaMorph software, the following parameters were quantified: 1) total nuclei (Hoechst-positive), 2) total myocyte nuclei (Hoechst-positive in desmin-positive cells), and 3) number of TUNEL-positive myocyte nuclei (TUNEL + /Hoechst/desmin + ). For maximum specificity, a "positive" result (ie, apoptotic cardiomyocyte) was counted only when a TUNEL-positive signal (green) was co-localized to nuclear DNA (blue) that was located within a cardiomyocyte (red).

Activation of caspase- 3 was used as another index of proapoptotic signaling. Total LV protein was extracted by homogenizing 20 to $30 \mathrm{mg}$ of $\mathrm{LV}$ myocardium in $4^{\circ} \mathrm{C}$ lysis buffer A (consisting of $25 \mathrm{mmol} / \mathrm{L}$ HEPES [pH 7.5], $10 \mathrm{mmol} / \mathrm{L} \mathrm{KCl}, 1.5 \mathrm{mmol} / \mathrm{L} \mathrm{MgCl}_{2}, 1 \mathrm{mmol} / \mathrm{L}$ ethylenediamine tetraacetic acid, $1 \mathrm{mmol} / \mathrm{L}$ ethylene glycol bis aminoethyl ether tetra-acetic acid, $1 \mathrm{mmol} / \mathrm{L}$ benzamidine, $1 \mathrm{mmol} / \mathrm{L}$ dithiothreitol, and $1 \mathrm{mmol} / \mathrm{L}$ phenylmethylsulfonyl fluoride) containing $1 \%[\mathrm{v} / \mathrm{v}]$ Triton X-100). After centrifugation at $100,000 \mathrm{~g}$ for 60 minutes at $4^{\circ} \mathrm{C}$, supernatant protein concentrations were determined using the Bradford method according to the manufacturer's instructions (BioRad, Hercules, Calif). Protein extract (25 $\mathrm{mg})$ and $25 \mathrm{~mL}$ reaction buffer $(0.1 \mathrm{~mol} / \mathrm{L}$ HEPES $\mathrm{pH} 7.0,10 \%$ polyethylene glycol, $0.1 \%$ cholamidopropyl dimethylammonio propanesulfonate, 10 $\mathrm{mmol} / \mathrm{L}$ dithiothreitol) were mixed with $1 \mathrm{~mL}$ of $5 \mathrm{mmol} / \mathrm{L}$ fluorogenic caspase-3-specific substrate (DEVD-AFC; BioMol, Plymouth Meeting, $\mathrm{Pa}$ ). After incubation at $37^{\circ} \mathrm{C}$ for 2 hours in 96-well fluorescence-specific plates, fluorescence was quantified in a microplate reader (Perkin Elmer, 
Boston, Mass) at excitation and emission wavelengths of 400 and $505 \mathrm{~nm}$, respectively. ${ }^{22}$ Relative caspase-3 activity was expressed as fold change relative to that found in LV from 8-week-old sham-operated animals.

\section{Assessment of Fibrosis}

Tissue sections were prepared as above, stained with Masson's trichrome, and imaged using light microscopy. Images were collected with a Leica digital color CCD-camera. Total image area and the relative percentage of collagen (blue) and tissue (red) were quantified using MetaMorph software.

\section{Estimation of Myocyte Number}

The total number of myocytes in the LV free wall of 7-week-old banded and sham-operated animals was estimated as described by Kajstura and colleagues. ${ }^{14}$ Briefly, this requires enzymatically dissociated for estimation of the frequency of mono-, bi-, and multinucleated myocytes (Hoechst staining) and confocal microscopic estimation of the cell volume of these myocyte populations by staining with FITC and propidium iodide (to label nuclei); cell area was computed and thickness was measured by optical sectioning in the $\mathrm{Z}$ plane. Next, the total ventricular volume was calculated as the weight of LV free wall myocardium per $1.06 \mathrm{~g} / \mathrm{mL}$, the specific gravity of muscle tissue. This was corrected for the percentage of replacement fibrosis (Masson's trichrome) to yield the volume of viable tissue, which was then used with the estimation of the volume fraction of myocytes in the viable tissue to yield the absolute volume of myocytes in the LV free wall. Next, the volume percentage of mono-, bi-, and multinucleated myocytes in the tissue was calculated, which in combination with the absolute volume of myocytes in the tissue allowed estimation of the aggregate volume of the different myocyte populations, and from this the number of myocytes in each population was estimated and summed to yield the total number of myocytes in the ventricle.

\section{Statistics \\ All results are expressed as mean \pm 1 standard deviation unless other- wise stated. To adjust for age- and growth-related changes in ventricular me- chanics, echocardiographic variables were expressed as Z-scores (normal deviates) relative to the distribution of each variable in the control animals. Comparison of groups over time was performed using 2-way analysis of variance (group and time) with repeated measures for time. Subsequent in- tergroup testing used Scheffe's method for post hoc pairwise comparisons. Fold changes in caspase- 3 activity between banded and age-matched sham controls were compared using the Wilcoxon rank-sum procedure. Compar- ison with the normal population (using Z-scores) was performed using a uni- variate $t$ test. Statistical analysis was performed on commercially available software (SAS Proc Mixed, SAS Institute Inc, Cary, NC).}

\section{RESULTS}

\section{Control Echocardiographic Data}

Thirteen normal rabbits were assessed serially from age 3 to 6 weeks during which time there was an approximate 4-fold increase in body mass. Heart rate, $\mathrm{SF}, \mathrm{VCFc}, \mathrm{SF}_{\mathrm{mw}}, \mathrm{VCFc}_{\mathrm{mw}}$, mass:volume ratio, systolic blood pressure, end-systolic and peak systolic stress, and end-systolic and peak FS were found to have no significant change over time. The progressive increase in LV end-diastolic dimension, posterior wall thickness, and body weight reflects the rapid growth seen in these animals in the first 7 weeks of life. Values from normal animals were used for comparison of banded animals with normal, aged-matched controls, as well as to describe the normal growth-related evolution of these variables. By using the measures of ESS obtained during afterload manipulation, the normative data for the VCFc-ESS (SVI), $\mathrm{SF}_{\mathrm{mw}}-\mathrm{FS}_{\mathrm{mw}}\left(\mathrm{SSI}_{\mathrm{mw}}\right)$, and $\mathrm{VCFc}_{\mathrm{mw}}-\mathrm{FS}_{\mathrm{mw}}\left(\mathrm{SVI}_{\mathrm{mw}}\right)$ relationships were determined. Table 1 summarizes these data over time.

\section{Serial Ventricular Mechanics after Aortic Banding}

No significant alteration in SF was noted at the time of aortic banding. The rate of increase in body weight was not different from that of controls. Detailed serial myocardial mechanics were available in 36 rabbits from weeks 3 to 7 and are summarized in Table 1 . The earliest detected change was an increase in LV mass:volume ratio (Figure 1), which was associated with a significant decrease in peak systolic stress compared with age-matched controls (Figure 2) by week 4 . Thereafter, mass:volume ratio progressively decreased, resulting in elevated peak systolic stress and reflecting a failure of adequate hypertrophy (Figures 1 and 2). Cardiac performance, as measured by SF and VCFc, declined progressively over time, reaching significantly lower levels by weeks 6 and 7. Similar patterns were seen with $\mathrm{SF}_{\mathrm{mw}}$ and $\mathrm{VCFc}_{\mathrm{mw}}$ reaching significantly lower levels by week 5. Myocyte contractility, as quantified by the SSI and SVI relationships, although initially sustained in the normal range, decreased at week 6 (Table 1). The use of midwall indices of contractility, which avoids the overestimation of cardiac performance seen with endocardial indices in hypertrophied myocardium, revealed significant changes. $\mathrm{SVI}_{\mathrm{mw}}$ and $\mathrm{SSI}_{\mathrm{mw}}$ decreased significantly by week 5 and continued to decline at 6 and 7 weeks. These serial changes reflect an initial period of adequate hypertrophy and later onset of inadequate hypertrophy and depression in myocardial contractility with concomitant chamber dilation.

\section{Cardiomyocyte Apoptosis}

Representative myocardial tissue sections with labeled nuclei (Hoechst-blue), myocytes (desmin-red), and nuclei undergoing DNA fragmentation (TUNEL-green) are shown in Figure 3, A. Quantification of TUNEL-positive cardiomyocytes in this model is shown in Figure $3, B$. The baseline number of TUNEL-positive nuclei ranged from 26 to 48 per million cardiomyocytes and did not change in the sham-operated animals. Most interestingly, a significant increase in myocyte apoptosis was apparent as early as 2 weeks after aortic banding ( 3-4 weeks of age); this was occurring at a time when LV mass was increasing, LV wall stress had normalized, and contractility was preserved (compare with Figures 1 and 2 and Table 1). There was a similar increase in LV tissue caspase- 3 activity at this early time point (Figure 4 ). The rate of myocyte apoptosis as indicated by TUNEL and activation of caspase-3 in the LV continued to increase, in association with inadequate hypertrophic compensation, increasing wall stress, decreasing contractility, and chamber dilatation.

\section{Left Ventricular Myocyte Number and Fibrosis}

There were no measurable differences in LV myocyte numbers within the sham-operated group; myocyte volume 
TABLE 1. Echocardiographic parameters in control and banded animals

\begin{tabular}{|c|c|c|c|c|c|c|c|c|c|c|}
\hline Variable & $\begin{array}{l}\text { Week } 3 \\
\text { Control }\end{array}$ & Banded & $\begin{array}{l}\text { Week } 4 \\
\text { Control }\end{array}$ & Banded & $\begin{array}{l}\text { Week } 5 \\
\text { Control }\end{array}$ & Banded & $\begin{array}{l}\text { Week } 6 \\
\text { Control }\end{array}$ & Banded & $\begin{array}{l}\text { Week } 7 \\
\text { Control }\end{array}$ & Banded \\
\hline SI (Z-score) & \pm 1.2 & $1 \pm 1.1$ & \pm 0.9 & \pm 0.9 & & $0.1 \pm 1.7$ & \pm 1.1 & $-1.0 \pm 2.0$ & $0.7 \pm 0.9$ & $-1.6 \pm 1.9^{\mathrm{a}}$ \\
\hline $\mathrm{SSI}_{\mathrm{mw}}$ (Z-score) & 0.8 & 0.6 & 0.9 & 1.0 & 0.9 & $-0.8 \pm 1.3^{\mathrm{a}}$ & 1.1 & $-1.3 \pm 1.6^{\mathrm{a}}$ & 1.2 & $-1.4 \pm 1.0^{\mathrm{a}}$ \\
\hline SVI (Z-score) & $0.2 \pm 1.1$ & $-0.4 \pm 0.2$ & $0.4 \pm 0.8$ & $-0.6 \pm 0.6$ & $0.4 \pm 0.6$ & $-1.2 \pm 0.2^{\mathrm{a}}$ & $0.6 \pm 1.2$ & $-1.6 \pm 0.4^{\mathrm{a}}$ & $0.6 \pm 0.9$ & $-1.7 \pm 0.5^{\mathrm{a}}$ \\
\hline $\mathrm{SVI}_{\mathrm{mw}}(\mathrm{Z}-\mathrm{sc}$ & $0.2 \pm 0.7$ & $0.2 \pm$ & $.2 \pm 1.6$ & $-0.6=$ & 0.4 & $-1.1 \pm$ & 1.4 & $-1.4 \pm 1.2^{\mathrm{a}}$ & 1.2 & -1.6 \\
\hline $\mathrm{SF}(\%)$ & $36.0 \pm 6.0$ & $34.2 \pm 6.0$ & $34.7 \pm 4.2$ & $37.0 \pm 5.5$ & $32.4 \pm 4.2$ & $32.9 \pm 13.5$ & $36.6 \pm 8.3$ & $24.1 \pm 14.9$ & $37.3 \pm 5.5$ & $15.1 \pm 11.5^{\mathrm{a}}$ \\
\hline $\mathrm{SF}_{\mathrm{mw}}(\%)$ & 3.1 & $21.1=$ & $19.5 \pm 3.8$ & 18.0 & $15.8 \pm 3.2$ & $14.8 \pm 6.4$ & 5.0 & $11.8 \pm 7.7^{\mathrm{a}}$ & $20.9 \pm 4.3$ & $8.6 \pm 6.8^{\mathrm{a}}$ \\
\hline $\mathrm{VCFc}(\mathrm{circ} / \mathrm{sec})$ & $1.8 \pm 0.4$ & $1.6 \pm 0.4$ & $1.7 \pm 0.3$ & $1.8 \pm 0.5$ & $1.6 \pm 0.2$ & $1.6 \pm 0.7$ & $1.8 \pm 0.5$ & $1.1 \pm 0.7$ & $1.8 \pm 0.3$ & $0.7 \pm 0.5$ \\
\hline $\mathrm{VCFc}_{\mathrm{mw}}(\mathrm{circ} / \mathrm{sec})$ & $1.0 \pm 0.2$ & $1.0 \pm 0.3$ & $1.0 \pm 0.2$ & $0.9 \pm 0.2$ & $0.8 \pm 0.2$ & $0.7 \pm 0.3$ & $1.1 \pm 0.3$ & $0.5 \pm 0.3$ & $1.0 \pm 0.2$ & $0.4 \pm 0.2$ \\
\hline Heart rate (beats/min) & $284 \pm 32$ & $292 \pm 37$ & $309 \pm 47$ & $299 \pm 42$ & $310 \pm 25$ & $305 \pm 37$ & $281 \pm 42$ & $306 \pm 42$ & $286 \pm 31$ & $293 \pm 33$ \\
\hline $\mathrm{SBP}(\mathrm{mm} \mathrm{Hg})$ & $80 \pm 19$ & $98.3 \pm 22.5$ & $83 \pm 30$ & $89.8 \pm 17.5$ & $83 \pm 13$ & $108.5 \pm 14.5$ & $89 \pm 13$ & $107.7 \pm 26$ & $87 \pm 11$ & $107 \pm 24.7$ \\
\hline LVEDD $(\mathrm{cm})$ & $0.8 \pm 0.1$ & $0.79 \pm 0.12$ & $0.9 \pm 0.1$ & $0.84 \pm 0.13$ & $1.0 \pm 0.1$ & $1.03 \pm 0.31$ & $1.0 \pm 0.2$ & $1.28 \pm 0.42$ & $1.0 \pm 0.2$ & $1.57 \pm 0.57^{\mathrm{a}}$ \\
\hline Body weight $(\mathrm{kg})$ & $0.35 \pm 0.28$ & $0.34 \pm 0.05$ & $0.54 \pm 0.46$ & $0.61 \pm 0.08$ & $0.86 \pm 0.20$ & $0.81 \pm 0.12$ & $1.33 \pm 0.24$ & $1.15 \pm 0.22$ & $1.59 \pm 0.48$ & $1.34 \pm 0.38$ \\
\hline
\end{tabular}

$L V$, Left ventricular; $S F$, shortening fraction; $S F_{m w}$, midwall shortening fraction; $V C F c$, rate of circumferential fiber shortening; $V C F c_{m w}$, midwall equivalent of rate of circumferential fiber shortening; $S S I$, stress shortening index; $S S I_{m w}$, midwall equivalent of stress shortening index; $S V I$, stress velocity index; $S V I_{m w}$, midwall equivalent of stress velocity index; $S B P$, systolic blood pressure; $L V E D D$, left ventricular end-diastolic dimension. ${ }^{a} P<.05$ compared with age-matched sham operated controls by Kruskal-Wallis (Z-scores and SF) or analysis of variance (SF, VCFc, SBP, LVEDD). Echocardiographic indices in normal sham-operated rabbits measured at 3 to 7 weeks of age. Data are mean \pm standard deviation, $\mathrm{n}=13$ each.

at 7 weeks of age was $22 \% \pm 5 \%$ greater than at 3 weeks $(P<.05)$. Compared with age-matched sham-operated animals at 7 weeks of age, total LV myocyte number decreased by $18 \% \pm 4 \%(P<.05)$, and myocyte volume increased an additional $43 \% \pm 8 \%(P<.05)$ in banded animals.

The relative proportion of collagen and muscle tissue $n$ LV myocardium was assessed by Masson's trichrome staining. Collagen represented approximately $6 \% \pm 2 \%$ of LV myocardial area. This was not changed with increasing age. There was also no significant difference between late hypertrophy (7 weeks) and age-matched controls in the LV area taken up by collagen $(P>.1)$.

\section{DISCUSSION}

Improved surgical techniques and younger age of repair have improved outcome in congenital heart disease. Despite

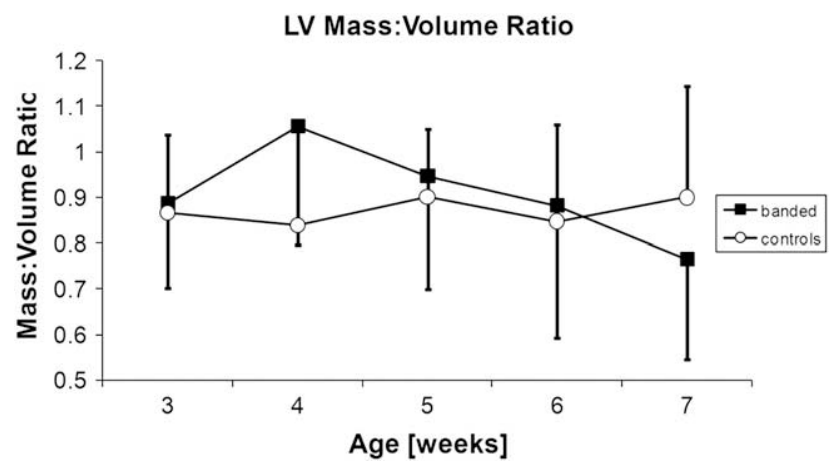

FIGURE 1. Effects of aortic banding of infant rabbits on LV mass:volume ratio determined by serial echocardiography. Data are mean \pm standard deviation $(\mathrm{SD}), \mathrm{n}=6-10$ each. $\# P<.05$ versus age-matched controls. $* P<.05$ versus 4 -week-old banded. LV mass:volume ratio increases early in hypertrophy and then progressively declines. $L V$, Left ventricular. advances in operative techniques and myocardial preservation methods, progressive ventricular dysfunction remains one of the most important problems that hamper long-term outcome in the treatment of congenital heart disease. A growing body of evidence indicates that apoptosis is responsible for some degree of myocyte loss and myocardial dysfunction resulting from hypoxia, ischemia, reperfusion, hypertrophy, heart failure, and inflammation. ${ }^{9-14,16,18}$ The major findings in the present study in the developing, pressure-loaded left ventricle are that significant myocyte apoptosis 1) begins with the onset of abnormal loading, 2) is not attenuated by normalization of wall stress during the period of compensatory hypertrophy, and 3) is sufficient to result in a measurable decrease in cardiomyocyte number as the ventricular dysfunction progresses to failure.

Chronic pressure overload-induced failure of the adult heart has long been suspected to be related to exacerbated

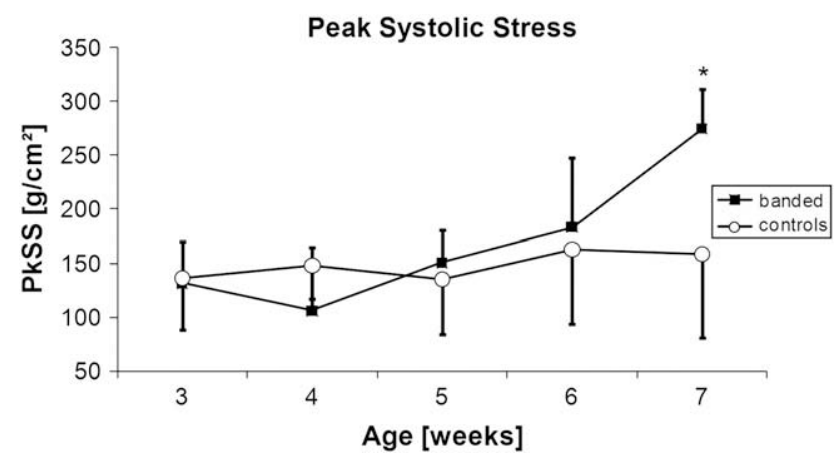

FIGURE 2. Effects of aortic banding of infant rabbits on LV peak systolic stress. Data are mean $\pm \mathrm{SD}, \mathrm{n}=6-10$ each. Peak systolic stress is reduced in banded animals at 4 weeks in association with increased LV mass (see Figure 1). Failure of continued hypertrophy in the face of increasing afterload results in a progressive increase in peak systolic stress. $* P<.05$ versus controls. PkSS, Peak systolic stress. 

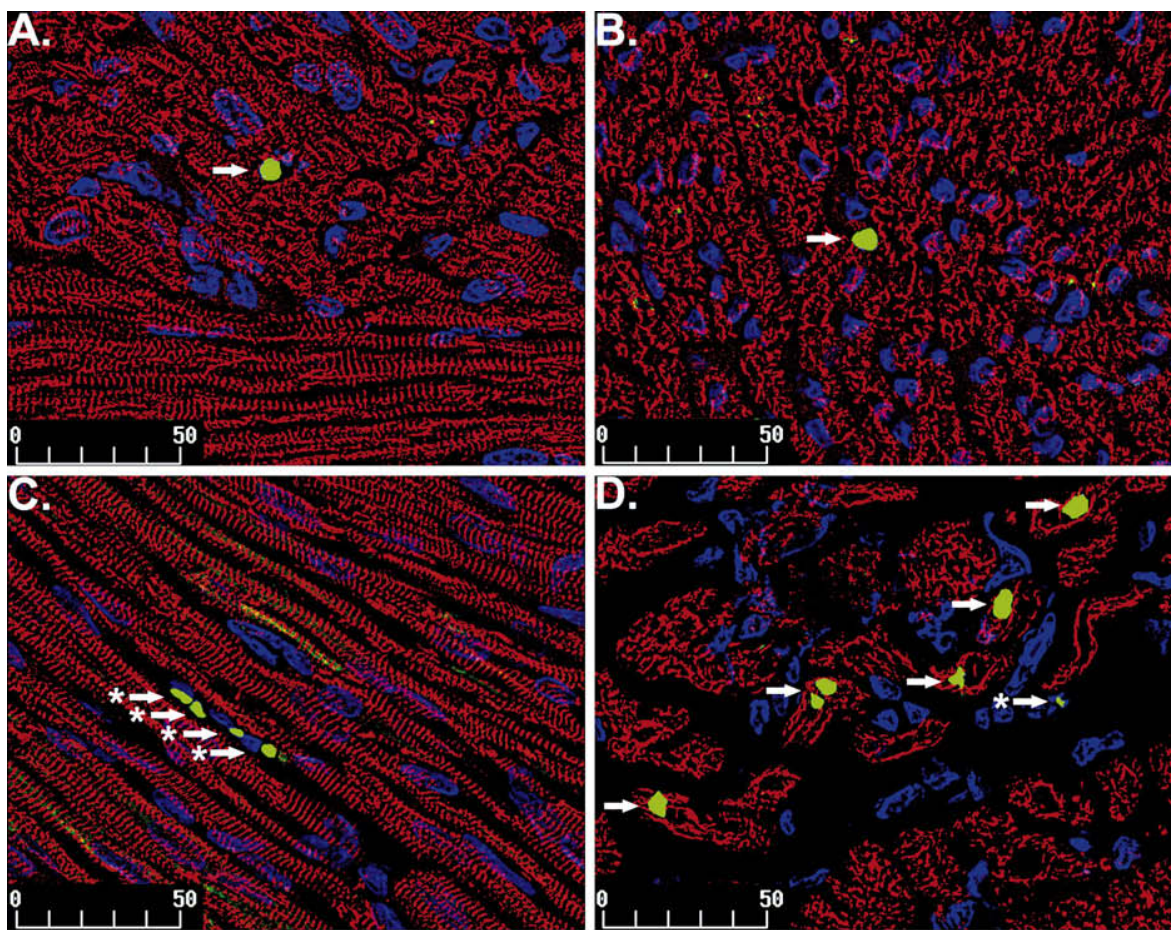

A

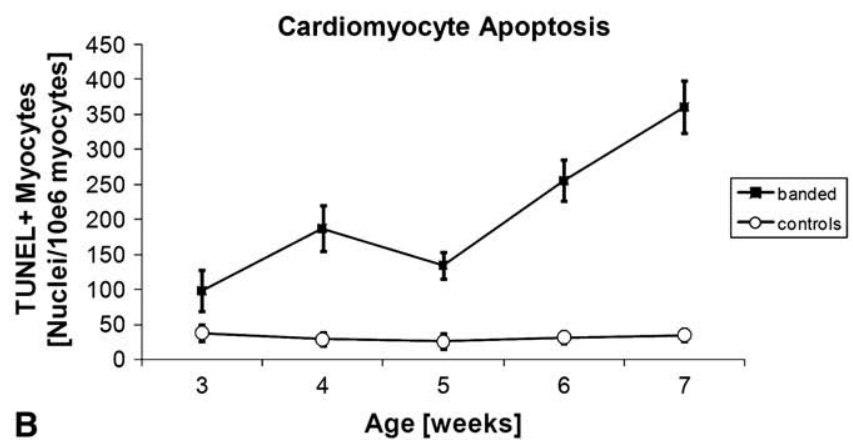

FIGURE 3. Apoptosis in LV myocardium of control sham-operated and banded infant rabbits. A, Representative fluorescent photomicrographs of 5- $\mu \mathrm{m}$ sections of the LV wall from banded rabbits. Sections were labeled with Hoechst to stain nuclear DNA (blue), desmin to identify cardiomyocytes (red), and DNA strand breaks (TUNEL-green). After image acquisition, images were subjected to 2-dimensional "no neighbors" deconvolution using a kernel that estimates the 3-dimensional point spread function based on the optics and imaging system. A and B, Banded animal at 3 weeks. C and D, Banded animal at 7 weeks. Apoptotic nuclei localized to myocytes (arrows); TUNEL-positive staining in non-myocytes (asterisks). B, Assessment of myocyte apoptosis in sham versus banded animals using this approach. Data are mean $\pm \mathrm{SD}, \mathrm{n}=6-8$ each. The difference between sham-operated and banded animals was significant at all ages.

cardiomyocyte apoptosis. ${ }^{23}$ This link has been demonstrated in several experimental models, ${ }^{21,22}$ and mitochondrial proteins (eg, apoptosis-inducing factor) and myogenic transcriptional regulators (eg, MyoD) have been recently implicated in the molecular mechanism. ${ }^{24,25}$ However, neither the time course nor the interrelation of cardiomyocyte apoptosis with the intricate changes in in vivo myocardial mechanics in response to pressure overload has been systematically analyzed. Moreover, the developing infant heart is likely to respond to pressure overload in a manner that is fundamentally different from the one observed in adult myocardium. The amount of myocyte apoptosis found in the present study is quantitatively similar to that measured in adult studies of pacing-induced heart failure, diabetes, and ischemic cardiomyopathy ${ }^{14,17}$ However, the degree to which apoptotic myocyte loss contributes to myocardial dysfunction and pathologic remodeling in any of these states remains uncertain. The effects of myocyte loss on ventricular function in the growing ventricle are likely to be at least as great as in the adult because of the significant increases in cardiac size and function that must occur to permit body growth. The physiologic remodeling and hypertrophy that accompany normal growth require maintaining an adequate number of myocytes, which are generally believed to be 


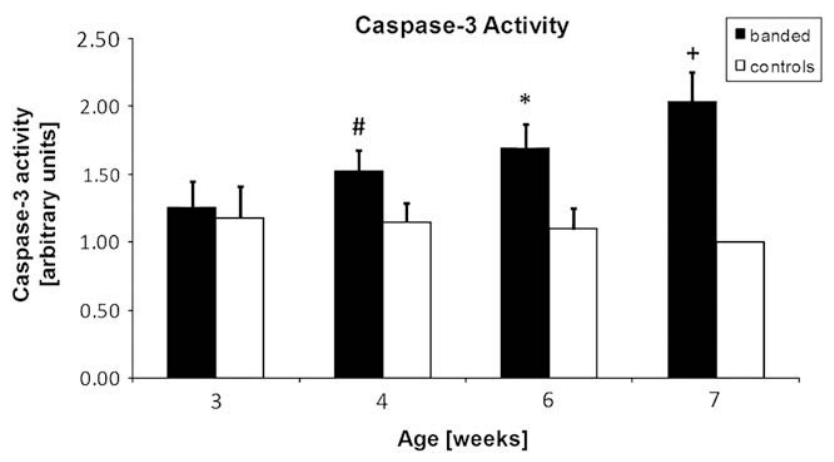

FIGURE 4. LV myocardial caspase-3 activity measured by cleavage of the specific fluorescent substrate DEVD-AFC (BioMol, Plymouth Meeting, Pa) in age-matched sham operated control (open bars) and banded hypertrophying (solid bars) hearts. Data are mean $\pm \mathrm{SD}, \mathrm{n}=5-7$ per group and expressed as arbitrary fluorescent units relative to that measured in 7-week controls. ${ }^{*} P=.04 . \# P=.03 .+P=.008$.

terminally differentiated and thus incapable of significant replacement. However, given the recent appreciation of the potential for cardiomyocyte regeneration by cell cycle reentry or via resident myocardial precursor cells, studies of myocyte proliferation as a possible mechanism to compensate for the hypertrophy-induced cell loss are clearly warranted.

The sensing and transduction mechanisms by which abnormal load is perceived and leads to the hypertrophic response remain uncertain, as are specific mechanisms responsible for myocyte apoptosis during hypertrophy. Current work favors connections between the myocyte cytoskeleton and the extracellular matrix, most likely focal adhesion kinases and integrins; signaling protein kinases within the extracellular matrix, altered sarcomeric function, altered substrate metabolism, ionic fluxes, and local neurohumoral mechanisms may also be involved in initiating the hypertrophic response. ${ }^{2}$ Downstream from the initiating signals, local production of cytokines, growth factors, catecholamines, and other compounds (eg, endothelin, angiotensin) stimulate myriad protein kinases and phosphatases, thereby promoting numerous quantitative and qualitative changes in gene expression. In many ways these responses recapitulate the fetal cardiomyocyte gene program and result in substantial alterations in cardiomyocyte signaling and functional pathways. ${ }^{2-6}$ The molecular events occurring during the hypertrophic response that could alter cell survival are numerous and include imbalances in cell survival and death pathways, such as those related to G-protein signaling, tumor necrosis factor- $\alpha$, phosphoinositide 3-kinase, calcineurin, and gp130, as well as altered calcium homeostasis, mitochondrial and substrate metabolism, and ischemia caused by insufficient capillary development. We found that apoptotic cell death is initiated during early compensatory hypertrophy and ultimately leads to a net loss of approximately $18 \%$ of the myocytes in our rabbit model.
In adult models, a low but significant frequency of cardiomyocyte apoptosis has been found in experimental hypertension and during the transition to failure (but not during adaptive compensation) in experimental aortic stenosis, pacing-induced heart failure, and humans with dilated cardiomyopathy and diabetes. ${ }^{10,14,16-18,22}$ In the present report, the presence of both cardiomyocyte-specific TUNEL staining and LV tissue caspase-3 activity represent relatively strong evidence that 1 or more proapoptotic programs are activated early in infant LV as it is responding to increased pressure loading. It is important to note that both results, although significant, are relatively modest in absolute quantitative terms compared with what has been reported in a variety of ischemia-reperfusion and other models. For example, 8-week-old rabbit left ventricles subjected to 20 minutes of normothermic ischemia followed by 2 hours of reperfusion demonstrated an approximate 7- to 12-fold increase in caspase- 3 activity (data not shown). In addition, these indices represent but a snapshot in time of a process that most likely is continuing and evolving over many days to weeks. Clearly, it would be useful to try to establish the quantitative relationship between the rate of apoptosis and the magnitude of ultimate cell loss. This computation, however, requires knowledge of the duration of TUNEL or other apoptotic signals in a cell that undergoes programmed cell death, which is not known and currently difficult or impossible to measure in in vivo models. However, there is increasing evidence that a relatively modest degree of apparent cardiomyocyte apoptosis similar to that observed in the present study can have progressive and deleterious contractile consequences over time. ${ }^{22,26}$ One recent study in sheep subjected to LV pressure overload also demonstrated onset of caspase activation during the apparent "compensated" phase of LV hypertrophy, which markedly increased during the development of LV failure. ${ }^{22}$ In terms of evolving contractile dysfunction, it is also important to note that caspase activation in cardiomyocytes can potentially result in the cleavage of contractile proteins (and thus contribute to the development of contractile dysfunction) independently of or in conjunction with effects leading to cell death. ${ }^{27}$

The present study is the first to identify an increase in apoptotic cardiomyocytes during the early phase of adaptive hypertrophy. This is an important finding because it suggests that normalizing wall stress in the absence of other interventions is not sufficient to attenuate proapoptotic signals active in pressure-loaded myocardium. This result is consistent with recent evidence that normalizing wall stress is not essential to preserve contractile function and prevent ventricular dilatation in chronic pressure overload. ${ }^{6,28}$

\section{Study Limitations}

There are several important limitations to the present study: TUNEL staining has well-known difficulties, 
particularly in terms of specificity. However, the microscopic method used did identify apoptotic myocytes with known sensitivity and specificity that have been used and validated under similar circumstances by others. ${ }^{14,16,17}$ TUNEL and other methods that detect DNA damage can show false-positive results, particularly in settings of necrosis (eg, ischemia); however, the occurrence of ischemia and necrosis would seem to be unlikely in early and compensated stages of hypertrophy. Moreover, we were able to support the likelihood of increased apoptotic events using a sensitive and specific fluorescent substrate for caspase-3; unfortunately, when this assay is performed on whole LV tissue it cannot reveal the cell type(s) responsible for any measured increase in caspase activity. However, these results, when taken together with the subsequent measured decrease in myocyte number, strongly support the conclusion that substantial cardiomyocyte apoptosis was occurring in this model.

As noted above, the pathways involved in hypertrophyassociated programmed cell death are likely to be multiple and interwoven. A related issue that must be addressed in the future is whether the observed frequency of myocyte apoptosis and reduced number of cardiomyocytes are important causes of the progression to failure that should be targeted for inhibition or instead is facilitating the controlled and beneficial (as opposed to necrosis, cell lysis, and inflammation) removal of damaged and dysfunctional myocytes and is therefore protective. It should also be emphasized that our findings cannot be directly translated to the setting of pressure-overload hypertrophy in the adult heart. In our model, the animal slowly grows into the aortic band, developing progressive constriction. These kinetics cannot be mimicked in adult rabbits. Similarly, the situation in manifest end-stage heart failure is probably different from that during compensated hypertrophy, but this was not a focus of the present study.

\section{CONCLUSIONS}

This study indicated that myocyte apoptosis is induced by pressure overload in the infant myocardium and is not attenuated during the period of compensated hypertrophy and normalized wall stress. From a clinical standpoint, these data suggest the pressure load, even at its earliest stages, is not tolerated by the developing left ventricle. They therefore support early and complete hemodynamic correction in the infant whenever possible.

\section{References}

1. Grossman W, Jones D, McLaurin LP. Wall stress and patterns of hypertrophy in the human left ventricle. J Clin Invest. 1975;56:56-64.

2. Lorell BH, Carabello BA. Left ventricular hypertrophy: pathogenesis, detection, and prognosis. Circulation. 2000;102:470-9.

3. MacLellan WR, Schneider MD. Genetic dissection of cardiac growth control pathways. Annu Rev Physiol. 2000;62:289-319.

4. Molkentin JD, Dorn IG 2nd. Cytoplasmic signaling pathways that regulate cardiac hypertrophy. Annu Rev Physiol. 2001;63:391-426.
5. Sadoshima J, Izumo S. The cellular and molecular response of cardiac myocytes to mechanical stress. Annu Rev Physiol. 1997;59:551-71.

6. Sano M, Schneider MD. Still stressed out but doing fine: normalization of wall stress is superfluous to maintaining cardiac function in chronic pressure overload. Circulation. 2002;105:8-10.

7. Tamai J, Hori M, Kagiya T, Iwakura K, Iwai K, Kitabatake A, et al. Role of alpha 1-adrenoceptor activity in progression of cardiac hypertrophy in guinea pig hearts with pressure overload. Cardiovasc Res. 1989;23:315-22.

8. Teiger E, Than VD, Richard L, Wisnewsky C, Tea BS, Gaboury L, et al. Apoptosis in pressure overload-induced heart hypertrophy in the rat. J Clin Invest. 1996; 97:2891-7.

9. Anversa P, Olivetti G, Leri A, Liu Y, Kajstura J. Myocyte cell death and ventricular remodeling. Curr Opin Nephrol Hypertens. 1997;6:169-76.

10. Ding B, Price RL, Goldsmith EC, Borg TK, Yan X, Douglas PS, et al. Left ventricular hypertrophy in ascending aortic stenosis mice: anoikis and the progression to early failure. Circulation. 2000;101:2854-62.

11. Gottlieb RA, Burleson KO, Kloner RA, Babior BM, Engler RL. Reperfusion injury induces apoptosis in rabbit cardiomyocytes. J Clin Invest. 1994;94: 1621-8.

12. Kajstura J, Cheng W, Reiss K, Clark WA, Sonnenblick EH, Krajewski S, et al. Apoptotic and necrotic myocyte cell deaths are independent contributing variables of infarct size in rats. Lab Invest. 1996;74:86-107.

13. Kajstura J, Cheng W, Sarangarajan R, Li P, Li B, Nitahara JA, et al. Necrotic and apoptotic myocyte cell death in the aging heart of Fischer 344 rats. Am J Physiol. 1996;271(3 Pt 2):H1215-28.

14. Kajstura J, Zhang X, Liu Y, Szoke E, Cheng W, Olivetti G, et al. The cellular basis of pacing-induced dilated cardiomyopathy. Myocyte cell loss and myocyte cellular reactive hypertrophy. Circulation. 1995;92:2306-17.

15. Kumar D, Kirshenbaum L, Li T, Danelisen I, Singal P. Apoptosis in isolated adult cardiomyocytes exposed to adriamycin. Ann N Y Acad Sci. 1999;874: 156-68.

16. Narula J, Haider N, Virmani R, DiSalvo TG, Kolodgie FD, Hajjar RJ, et al. Apoptosis in myocytes in end-stage heart failure. N Engl J Med. 1996;335:1182-9.

17. Olivetti G, Abbi R, Quaini F, Kajstura J, Cheng W, Nitahara JA, et al. Apoptosis in the failing human heart. N Engl J Med. 1997;336:1131-41.

18. Olivetti G, Quaini F, Sala R, Lagrasta C, Corradi D, Bonacina E, et al. Acute myocardial infarction in humans is associated with activation of programmed myocyte cell death in the surviving portion of the heart. J Mol Cell Cardiol. 1996;28: 2005-16.

19. Thompson CB. Apoptosis in the pathogenesis and treatment of disease. Science. 1995;267:1456-62.

20. Condorelli G, Morisco C, Stassi G, Notte A, Farina F, Sgaramella G, et al. Increased cardiomyocyte apoptosis and changes in proapoptotic and antiapoptotic genes bax and bcl-2 during left ventricular adaptations to chronic pressure overload in the rat. Circulation. 1999;99:3071-8.

21. Li Z, Bing OH, Long X, Robinson KG, Lakatta EG. Increased cardiomyocyte apoptosis during the transition to heart failure in the spontaneously hypertensive rat. Am J Physiol. 1997;272(5 Pt 2):H2313-9.

22. Moorjani N, Ahmad M, Catarino P, Brittin R, Trabzuni D, Al-Mohanna F, et al. Activation of apoptotic caspase cascade during the transition to pressure overloadinduced heart failure. J Am Coll Cardiol. 2006;48:1451-8.

23. Bing $\mathrm{OH}$. Hypothesis: apoptosis may be a mechanism for the transition to heart failure with chronic pressure overload. J Mol Cell Cardiol. 1994;26: 943-8.

24. Ha T, Hua F, Li Y, Ma J, Gao X, Kelley J, et al. Blockade of MyD88 attenuates cardiac hypertrophy and decreases cardiac myocyte apoptosis in pressure overload induced cardiac hypertrophy in vivo. Am J Physiol Heart Circ Physiol. 2006;290:H985-94. Epub 2005 Sep 30.

25. van Empel VP, Bertrand AT, van der Nagel R, Kostin S, Doevendans PA, Crijns HJ, et al. Downregulation of apoptosis-inducing factor in harlequin mutant mice sensitizes the myocardium to oxidative stress-related cell death and pressure overload-induced decompensation. Circ Res. 2005;96:e92-101.

26. Foo RS, Mani K, Kitsis RN. Death begets failure in the heart. J Clin Invest. 2005; 115:565-71.

27. Communal C, Sumandea M, de Tombe P, Narula J, Solaro RJ, Hajjar RJ. Functional consequences of caspase activation in cardiac myocytes. Proc Natl Acad Sci U S A. 2002;99:6252-6.

28. Esposito G, Rapacciuolo A, Naga Prasad SV, Takaoka H, Thomas SA, Koch WJ, et al. Genetic alterations that inhibit in vivo pressure-overload hypertrophy prevent cardiac dysfunction despite increased wall stress. Circulation. 2002;105: $85-92$. 


\section{Appendix. Echocardiographic methods}

\section{Blood Pressure}

Determination of the accuracy of optical plethysmography was performed on 8 occasions in rabbits aged 4 to 6 weeks. After anesthesia was induced with intramuscular ketamine $(20 \mathrm{mg} / \mathrm{kg})$ and xylazine $(0.5 \mathrm{mg} / \mathrm{kg})$, the right upper limb was shaved and topical alcohol was applied. Dissection of the neck vessels and direct cannulation of the carotid artery were achieved using a 24-gauge polyethylene catheter. A 2F high-fidelity (Millar, Houston, Tex) catheter was inserted in line with the fluid-filled catheter. The central arterial waveform was captured digitally using a standard analog-to-digital converter at a sampling rate of $200 \mathrm{~Hz}$. Simultaneous right upper limb brachial arterial blood pressure was determined using optical plethysmography (Finapress, Ohmeda, Englewood, Colo). The Finapress was applied to the shaved right forearm after application of topical alcohol over the region of the palpable brachial artery.

Comparison of the noninvasive arterial waveform with the high-fidelity direct central measurement was performed using customized software. Beat-to-beat waveform segmentation was followed by peak and trough detection to calculate systolic and diastolic values. Determination of the variation (bias) of optical plethysmography compared with Millar measurement was expressed as the mean and standard deviation (SD) of the difference between noninvasive and invasive values. To estimate the magnitude of error (accuracy) the mean \pm SD of the absolute differences was calculated. To quantify the magnitude of error in relationship to derived variables of stress, a comparison of Finapress-derived versus Millar-derived ESS and peak systolic stress was also performed. The bias, accuracy, and mean percentage absolute error were then calculated.

Spectral analysis of power and frequency components was performed on both the noninvasive and invasive arterial waveforms. Spectral analysis was performed using a nonparametric spectral estimation technique based on a discrete Fourier transform. Specifically, a modified averaged periodogram method was used to decrease the high variance incurred with direct application of the discrete Fourier transform. A 30-second data segment was used for power spectral analysis. Transfer function (magnitude and phase) and power spectrum were assessed.

A comparison of the direct high-fidelity central arterial systolic and diastolic blood pressure and those recorded by Finapress was determined. The noninvasive waveform revealed a narrower pulse pressure and generally higher diastolic blood pressure. Bias as measured by mean and $1 \mathrm{SD}$ for the error was $-1.1 \pm 4.0$ and $0.85 \pm 3.2 \mathrm{~mm} \mathrm{Hg}$ for systolic and diastolic blood pressures, respectively. Accuracy, the absolute mean, and SD of the error was $5.9 \pm 4.7$ and $9.2 \pm 6.9 \mathrm{~mm} \mathrm{Hg}$ for systolic and diastolic blood pressures, respectively.
Fourier analysis revealed similar frequency components for both Millar and Finapress waveforms. A similar time course of the pressure waveforms was documented, supported by the transfer magnitude approximating one. Noninvasive waveform contains less power at the beat frequency and its harmonics, suggesting that it does underestimate the pulse pressure. A phase lag consistent with the more peripheral measurement of the Finapress was noted.

To better appreciate the error incurred using the Finapress blood pressure in the determination of derived stress indices, calculation of derived myocardial indices at the time of invasive versus noninvasive blood pressure comparison was performed. The noninvasively derived indices were compared with invasively derived indices using the same measures of chamber size and wall thickness. Overall, there was a small underrepresentation of both stress measures (end systolic and peak systolic). Bias was $-4.6 \pm 8.8$ and -10.5 $\pm 16.3 \mathrm{~g} / \mathrm{m}^{2}$ and accuracy was $6.95 \pm 7.1$ and $14.1 \pm$ $13.2 \mathrm{~g} / \mathrm{m}^{2}$ for end-systolic and peak systolic stress, respectively. The mean percentage absolute error was $11.0 \pm$ $7.4 \%$ for ESS and $8.15 \pm 4.9 \%$ for peak systolic stress.

\section{Echocardiography}

Weekly assessment of cardiac function was performed from the time of aortic banding. Noninvasive blood pressure assessment was limited by animal size and first measured at 3 weeks of age. All earlier studies were limited to 2-dimensional and M-mode echocardiographic assessment. Thereafter, weekly evaluations were performed using echocardiographic and optical plethysmography. Transthoracic echocardiography was performed using an Acuson 128 (Siemens USA, Malvern, Pa) or Hewlett-Packard Sonos 1500 Cardiac Imager (Philips Healthcare, Andover, Mass) equipped with a 7 to $7.5-\mathrm{MHz}$ transducer. Adequate ultrasound surface-interface coupling was achieved by initial shaving of the anterior surface of the thorax and application of ultrasound gel. All imaging was performed under light restraint without sedation. Pulsed Doppler interrogation of the LV outflow tract from an apical view with simultaneous electrocardiogram was recorded at high-speed $(100 \mathrm{~mm} / \mathrm{s})$, hard-copy printout from the strip chart recorder for determination of ejection time. Two-dimensional crosssectional imaging and M-mode interrogation of the LV short axis at the level of the papillary muscles were performed. High-speed (100 mm/s), hard-copy, 2-dimensionally directed echocardiographic M-mode recordings of the LV minor axis were recorded simultaneously with electrocardiogram and noninvasive peripheral arterial pulse waveform using optical plethysmography (Finapress).

Determination of the normal endocardial SSI and SVI relationships and their midwall equivalents $\left(\mathrm{SSI}_{\mathrm{mw}} \mathrm{SVI}_{\mathrm{mw}}\right)$ was undertaken in 13 control animals between weeks 3 and 7 . To determine the normal relationship of the function 
indices to afterload, assessment at resting afterload was followed by determination at increased afterload during atropine and methoxamine administration. The animals were premedicated with atropine, $0.01 \mathrm{mg} / \mathrm{kg}$ (minimum dose of $0.1 \mathrm{mg}$ ) intravenously, to maintain a stable heart rate during subsequent studies with repeat dosing as needed. After several stable recordings to determine baseline blood pressure and afterload, an intravenous infusion of methoxamine (25 $\mu \mathrm{g} / \mathrm{kg} / \mathrm{min}$ ) was begun. A gradual increase in systolic blood pressure to 36 to $60 \mathrm{~mm} \mathrm{Hg}$ above baseline was obtained. A total of 56 measurements were obtained.

\section{Analysis of Echocardiography}

The noninvasive arterial pulse tracing, LV endocardial border of the septum, and endocardial and epicardial borders of the posterior wall were hand digitized using a bit pad interfaced to a microcomputer-based digitizing station with custom software. This system is programmed to adjust the sampling rate of the tablet to $200 \mathrm{~Hz}$, which is adequate to obtain 20 to 25 nonaliased harmonics of heart rates of 240 to 300 beats $/ \mathrm{min}$. After data input, the pulse transmission delay is corrected by electronically aligning the pulse upstroke to the time of the onset of ejection as determined from the LV outflow Doppler sample. End systole was defined as the time of cessation of antegrade flow as determined from the LV outflow track Doppler signal. The time of end systole was used to ascertain end-systolic pressure from the blood pressure recording.

From the digitized data, the following measurements were obtained by averaging 3 cardiac cycles: 1) pressure during LV ejection, calculated by assignment of diastolic pressure to the minimum and systolic pressure to the maximum of the pulse tracing; 2) LV internal diameter; 3) LV posterior wall thickness; 4) LV midwall dimensions by the method of Shimizu and colleagues; 5) LV meridional wall stress in grams/centimeters squared, calculated throughout ejection according to Grossman and colleagues: ${ }^{1}$

systolic stress $=\frac{P \times D \times 1.35}{h \times\left(1+\frac{h}{D}\right) \times 4}$

where $\mathrm{P}$ is the LV pressure in millimeters of mercury, $\mathrm{D}$ is the $\mathrm{LV}$ endocardial cross-sectional diameter in centimeters, $\mathrm{h}$ is the posterior wall thickness in centimeters, and 1.35 is the conversion factor from $\mathrm{mm} \mathrm{Hg}$ to grams/centimeters squared, and ventricular FS is according to the equation of Regen:

$F S=\frac{1.35 \times P\left(\frac{h}{\ln (D+h)}-\ln D\right)}{2 \times h}$

From the continuous data, end-diastolic values for shortaxis dimension and wall thickness were taken at the time of maximal LV dimension, and end-systolic values for shortaxis dimension, wall thickness, blood pressure, meridional wall stress, and FS were determined at the time of end systole on the Doppler tracing. Meridional peak systolic stress and FS were obtained from the continuous systolic stress calcultation. Similar calculations were performed for peak and end-systolic FS. LV ejection time was measured from the LV outflow Doppler tracing and adjusted to the heart rate of 60 beats/min by dividing by the square root of the R-R interval on the electrocardiogram. The LV circumferential fiber $\mathrm{SF}$ and midwall SF were calculated with the use of short-axis dimensions as (end-diastolic dimension - end-systolic dimension)/end-diastolic dimension. VCFc was calculated as the SF divided by the rate-adjusted ejection time. Midwall $\mathrm{VCF}$ c was similarly calculated using midwall equivalents. The LV mass:volume ratio was estimated from determination of LV epicardial and endocardial cross-sectional area as determined from M-mode measurements.

\section{Stress-velocity and Stress-shortening Indices}

The relation between rate-adjusted mean velocity of shortening and end-systolic meridional stress (SVI) has been shown to be an afterload-adjusted, preload-independent index of contractility whether obtained as a global or regional index. The relation is inversely linear, and in healthy humans the slope of the relationship for any one person is parallel to the slope of the relation for the total study group. The value of the SVI for each banded rabbit was determined relative to the calculated distribution of this index in normal subjects. The SVI is reported as a normal deviate (Z-score) relative to the distribution in normal subjects. Thus, the $\mathrm{SVI}=\left(\mathrm{X}_{1}-\mu\right) / \sigma$ where $\mathrm{X}_{1}=$ measured rate-adjusted mean velocity of circumferential fiber shortening, $\mu=$ the group mean rate-adjusted mean velocity of circumferential fiber shortening for the measured end-systolic meridional stress, and $\sigma=$ the standard error of the regression for the group stress-velocity index at that level of end-systolic meridional stress. An SVI of less than negative 2 (ie, 2 SD below the normal mean value) was considered to represent myocardial dysfunction. Similarly, the SSI is the relation of SF and ESS. This index of contractility is preload sensitive. The SSI is correspondingly quantified as the normal deviate of SF for the given ESS, obtained in a manner analogous to that described for the SVI. FS is stress independent of radial forces and was used relative to midwall cardiac performance measures $\left(\mathrm{SF}_{\mathrm{mw}}\right.$ and $\left.\mathrm{VCF}_{\mathrm{mw}}\right)$ to determine load-independent measures of contractility $\left(\mathrm{SSI}_{\mathrm{mw}}\right.$ and $\left.\mathrm{SVI}_{\mathrm{mw}}\right)$.

\section{Further Reading}

Shimizu G, Zile MR, Blaustein AS, Gaasch WH. Left ventricular chamber filling and midwall fiber lengthening in patients with left ventricular hypertrophy: overestimation of fiber velocities by conventional midwall measurements. Circulation. 1985; 71:266-72.

Regen DM. Calculation of left ventricular wall stress. Circ Res. 1990;67:245-52.

Colan SD, Borow KM, MacPherson D, Sanders SP. Use of the indirect axillary pulse tracing for noninvasive determination of ejection time, upstroke time, and left ventricular wall stress throughout ejection in infants and young children. Am J Cardiol. 1984;53:1154-8. 
Colan SD, Borow KM, Neumann A. Left ventricular end-systolic wall stress-velocity of fiber shortening relation: a load-independent index of myocardial contractility. J Am Coll Cardiol. 1984;4:715-24.

Colan SD, Sanders SP, Ingelfinger JR, Harmon W. Left ventricular mechanics and contractile state in children and young adults with end-stage renal disease: effect of dialysis and renal transplantation. J Am Coll Cardiol. 1987;10:1085-94.
Segar DS, Moran M, Ryan T. End-systolic regional wall stress-length and stressshortening relations in an experimental model of normal, ischemic and reperfused myocardium. J Am Coll Cardiol. 1991;17:1651-60.

Colan SD, Trowitzsch E, Wernovsky G, Sholler GF, Sanders SP, Castaneda AR. Myocardial performance after arterial switch operation for transposition of the great arteries with intact ventricular septum. Circulation. 1988;78:132-41. 